\title{
RECTANGULAR MICROWAVE PHOTONIC FILTER BASED ON STIMULATED BRILLOUIN SCATTERING IN FIBER
}

\author{
Lilin $\mathrm{Yi}^{1}$, Wei Wei ${ }^{1}$, Yves Jaouen ${ }^{2}$,Mengyue Shi ${ }^{1}$ and Weisheng $H u^{1}$
}

\author{
${ }^{1}$ State Key Lab of Advanced Optical Communication Systems and Networks, Shanghai Jiao Tong \\ University, Shanghai 200240, China \\ ${ }^{2}$ Institut Télécom, Télécom ParisTech, CNRS UMR 5141, 46 Rue Barrault, 75634 Paris, France \\ Email: lilinyi@sjtu.edu.cn
}

\begin{abstract}
We demonstrate a rectangular microwave photonic filter based stimulated Brillouin scattering effect in optical fiber. The rectangular spectral response is achieved by using both multi-tone and frequency-sweeping Brillouin pump schemes, combined with digital feedback compensation. The filter selectivity is improved by using pump-splitting dual-stage scheme. A rectangular microwave photonic filter with as high as 45-dB selectivity at 1-GHz bandwidth has been achieved.
\end{abstract}

Keywords: Microwave photonic filter, rectangular, stimulated Brillouin scattering

\section{INTRODUCTION}

In radio-frequency (RF) systems, there have been continuously interests to use photonic devices to implement flexible signal filtering function, which can be generally named as microwave photonic filter (MPF). An ideal MPF should have a single passband with flexible tenability on bandwidth, central frequency and selectivity. Most importantly, the MPF should have rectangular response with flat-top and sharp roll-off to select the desired microwave signals with high rejection of the out-of-band signals. MPFs have been generally realized by RF signal modulated single-frequency optical source followed by delayed tap filter [1] or RF signal modulated multi-wavelength optical source followed by dispersion element [2]. Optical comb has also been proposed to improve the tap numbers for enhanced filter configurability [3]. But ideal rectangular MPF with flexible tenability is extremely challenging. On the other hand, narrowband optical filter is a good candidate for MPF by mapping the microwave signals to the optical field then processing the signal in optical domain finally converting back to the microwave domain. Programmable optical processor based on liquid crystal on silicon (LCOS) [4] and virtually imaged phased-array (VIPA) [5] have been proposed to achieve narrowband flat-top MPF, but limited by the spectral resolution, ideal rectangular filter in $\sim \mathrm{GHz}$ region cannot be achieved.

Stimulated Brillouin scattering (SBS) in fiber has been proposed to act as an active optical filter. By controlling the pump spectrum using external phase modulation [6], direct current modulation [7] and cascaded phase and intensity modulation [8], the SBS gain spectrum can be spectral-shaped and broadened to several GHz. But rectangular SBS gain demonstration is very challenging since the steep edge is the most difficult part due to the gradual change characteristic of the pump spectrum at the edge. By shaping the SBS pump with digitally-controlled electrical multi-tone with a feedback compensation method, we have achieved a rectangular optical filter with bandwidth tuning from $50 \mathrm{MHz}$ to 3 $\mathrm{GHz}$ for the first time [9]. By implementing nonlinearity management to mitigate the four-wave mixing (FWM) effect of the ultra-dense pump lines and utilizing a more suitable fiber with a single Brillouin gain peak, the in-band ripple is decreased to $\sim 1 \mathrm{~dB}$ [10]. But electrical/optical nonlinearities originated from the multi-tone pumps generate undesired out-of-band gain meanwhile pump depletion limits the maximal signal gain therefore limiting the filter selectivity to around 15 $\mathrm{dB}$ for $2-\mathrm{GHz}$ bandwidth. By using pump-splitting dual-stage scheme to suppress the strong FWM effect of the multi-tone pump and pump depletion, the filter selectivity can be improved to $30 \mathrm{~dB}$ for $2-\mathrm{GHz}$ bandwidth [11]. To completely suppressing the out-of-band SBS gain, it is desired to use frequency-sweeping pump scheme [12] where no FWM effect happens. By implementing digital feedback compensation and pump-splitting dual-stage configuration for the frequency-sweeping scheme, the selectivity is improved to $45 \mathrm{~dB}$ for $2-\mathrm{GHz}$ bandwidth. The $20-\mathrm{dB}$ shape factor is 1.056 at $1-\mathrm{GHz}$ bandwidth, which is almost close to the ideal rectangular case, and is the best value among all the MPFs in $\sim \mathrm{GHz}$ bandwidth region, to the best of our knowledge [13].

\section{MULTI-TONE SBS PUMP BASED MPF DEMONSTRATION}

For multi-tone SBS pump based MPF, the filter flatness can be reduced to $1 \mathrm{~dB}$ for the bandwidth from $100 \mathrm{MHz}$ to $4 \mathrm{GHz}$ by implementing nonlinearity management and suitable fiber with a sing Brillouin peak [10]. The filter selectivity can be improved by increasing the pump power therefore the SBS gain. However there are some limitations on the SBS gain. Firstly when the Brillouin pump power is higher than several hundreds of $\mathrm{mW}$, it starts acting as Raman pump at the same time which results in SBS pump power consumption. The second limitation on the filter selectivity is FWM effect induced by multi-frequency comb. It generates new components 
out of the filter passband and cannot be completely mitigated by the nonlinearity management method. When the pump power increases, FWM effect is getting much more severe especially in large bandwidth cases. The out-of-band gain will consume considerable amount of pump power and disrupt the rectangular shape generation.

In order to increase the filter selectivity, we propose a pump-splitting dual-stage scheme. Instead of using a single pump with high power, we split it into two stages and amplify the signal twice successively. Note that the filter selectivity is only related to the on-off gain and has nothing to do with the transmission loss, extra attenuation can be induced between the 2 stages to prevent the signal saturation in the second stage. In this case, the pump power of each stage is not too high to stimulate high Raman gain nor to induce too much out-of-band FWM components. Moreover, the decrease in pump power for each stage will decrease the induced noise from the spontaneous Brillouin emission. Thus the filter selectivity can be increased dramatically and better noise performance can be achieved at the same time.


(iii)

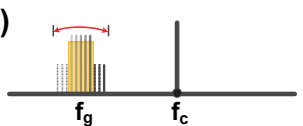

(iv)

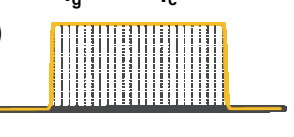

Fig. 1. Experimental setup. Inset (i) single sideband pump $f_{p}, S B S$ gain around $\mathrm{f}_{\mathrm{g}}$, and ECL laser frequency $\mathrm{f}_{\mathrm{c}}$, (ii) sweeping probe signal, (iii) sweeping probe signal amplified by the SBS gain, (iv) measured gain spectrum

The experimental setup for multi-tone SBS pump based MPF is shown in Fig. 1. A distributed feedback (DFB) laser operating at $\sim 1543 \mathrm{~nm}$ is split into two branches acting as pump and probe signal in the upper and lower branch respectively. In the upper branch, an arbitrary waveform generator (AWG) is used to generate the electrical spectral lines with random frequency interval within $\pm 1-\mathrm{MHz}$ deviation from the natural SBS bandwidth of $20 \mathrm{MHz}$, i.e. $19 \mathrm{MHz}, 20 \mathrm{MHz}$ and 21 $\mathrm{MHz}$. Then it modulates the $\mathrm{CW}$ light to generate the optical carrier-suppressed single-sideband (OCS-SSB) SBS pump lines utilizing an I\&Q modulator (IQM). After boosted by a high power EDAF, the OCS-SSB signal is then split into two parts equally and send into two $25 \mathrm{~km}$ long fiber. In each stage, a polarization controller (PC) is used to maintain the SBS gain at the maximum value. The SBS gain is $\sim 11-\mathrm{GHz}$ away from the pump as shown in Fig. 1(i). In the lower branch, a sweeping signal covering the whole SBS gain region from an electrical vector network analyzer (EVNA) modulates the $\mathrm{CW}$ light to generate the probe signal. After removed the left sideband by an optical bandpass filter (BPF) shown in Fig. 1(ii), the probe light goes through the 2 fibers successively and is amplified once it sweeps within the SBS gain region as shown in Fig. 1(iii). An optical attenuator between the two stages can effectively increase the filter selectivity and prevent pump depletion in the second stage. The probe signal is then detected by a photodiode (PD) and sent into the EVNA. The amplitude response sketched in Fig. 1(iv) are measured by the EVNA and the SBS gain spectrum can be obtained by subtracting the results with and without the SBS amplification.

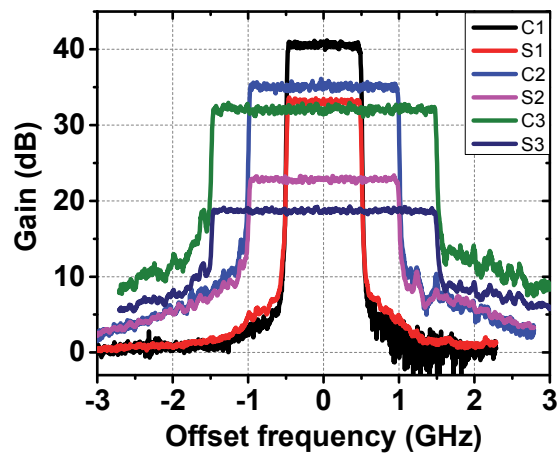

Fig. 2. Filter comparison between single- and dual-stage approaches. C: cascaded-stage; S: single-stage.

The spectral responses of both single- and dual-stage filters are shown in Fig. 2. The pump power is set to 21, 24 and $27 \mathrm{dBm}$ for filter bandwidth of 1, 2 and $3 \mathrm{GHz}$ respectively. As shown in Fig. 2, for all bandwidth cases, the filter gain has been improved obviously with the same total pump power while the passband flatness remains at the same level. The larger the bandwidth is, the larger the increase is. For the $3-\mathrm{GHz}$ filter, the maximum gain of single-stage filter is limited to $\sim 18.5 \mathrm{~dB}$ due to SRS and the severe out-of-band FWM components. By using the dual-stage scheme, the gain has been increased to $\sim 32 \mathrm{~dB}$. The high selectivity makes the filter shape more like rectangular than flat-top trapezoid. For the $1-\mathrm{GHz}$ dual-stage filter, by using dual-stage pump not only is the gain increased, but also the out-of-band FWM effect is slightly mitigated. The results have clearly proved the validity of the dual-stage scheme to increase the filter selectivity.

\section{FREQUENCY-SWEEPING SBS PUMP BASED MPF DEMONSTRATION}

For the multi-tone SBS pump based MPF, the out-of-band SBS gain originated from the FWM effect of multiple pump lines limits the filter selectivity. The out-of-band SBS gain can be completely suppressed for frequency-sweeping SBS pump scheme since there is only a single frequency existed at any specific time therefore no FWM effect happens. The experimental 
setup of frequency-sweeping SBS pump based MPF is shown in Fig. 3. Compared with the multi-tone SBS pump based MPF in Fig. 1, the main difference of the scheme is that the AWG is used to generate the frequency sweeping signal rather than multi-tone signal. Besides two laser sources serve as the Brillouin pump and probe respectively therefore the central frequency of the MPF can be flexibly tuned by changing the pump and probe wavelength difference. The waveform generated by AWG and the pump power determine the shape and selectivity of the filter, respectively. Digital feedback compensation scheme is implemented to achieve the desired SBS gain spectrum. The frequency sweeping time is a key parameter to achieve a flat response after feedback compensation. Fast sweeping in $\sim$ ns duration will make the feedback algorithm fails. Slow sweeping in $\sim$ us duration can achieve very flat response but the sweeping duration should shorter than the signal propagation time in the fiber therefore the signal wave is subjected to SBS amplification by the entire sweeping pump spectrum. In the experiment, we fix the frequency sweeping duration at 1 us, which can achieve effective feedback compensation and is also much shorter than the propagation time of $\sim 120$ us in 25-km SMF.

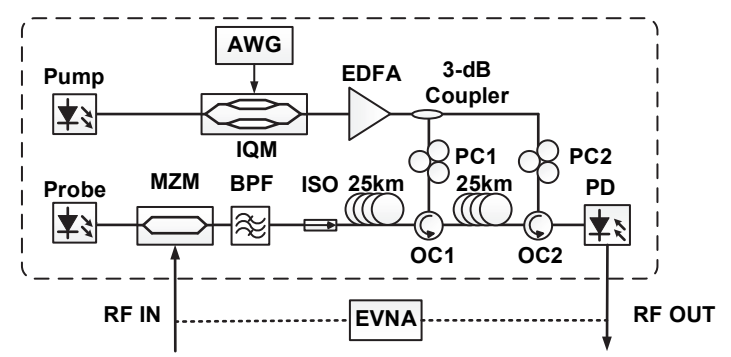

Fig. 3. Experimental setup of frequency-sweeping SBS pump based MPF
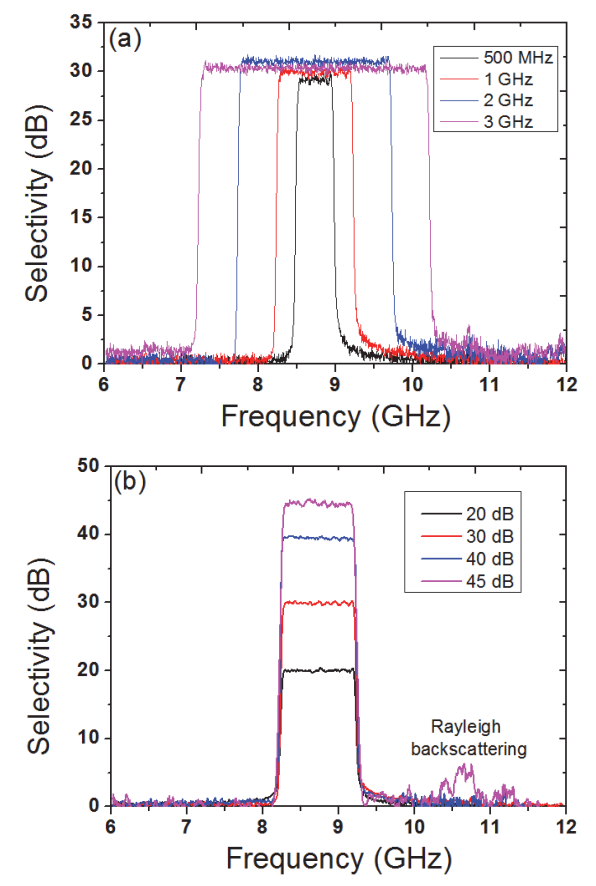

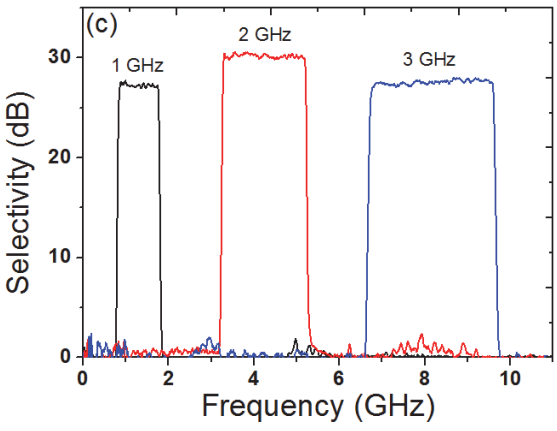

Fig. 4. Filter bandwidth, selectivity and central frequency tuning demonstration for frequency-sweeping pump scheme.

By changing the bandwidth of the sweeping pump signal, the filter bandwidth can be tuned from $500 \mathrm{MHz}$ to $3 \mathrm{GHz}$ while keeping the passband ripple less than $+/$ $0.7 \mathrm{~dB}$ as shown in Fig. 4 (a). The filter selectivity can be tuned by simply changing the pump power with the same pump waveform. Take a $1-\mathrm{GHz}$ rectangular filter as an example, the selectivity can be increased from $20 \mathrm{~dB}$ to $45 \mathrm{~dB}$ by increasing the pump power from $18.2 \mathrm{dBm}$ to $23.2 \mathrm{dBm}$, while keeping the ideal rectangular shape as shown in Fig. 4 (b). By tuning the pump wavelength, the central frequency of the filter can be flexibly tuned while the rectangular shape still keeps unchanged. Simultaneously, the filter bandwidth can be tuned by modifying the pump waveform stored in the memory as shown in Fig. 4(c).

\section{CONCLUSION}

We have presented a rectangular MPF based on stimulated Brillouin scattering in optical fiber. The rectangular spectral response is achieved by using both multi-tone pump and frequency-sweeping pump schemes. The filter passband flatness is guaranteed by using feedback compensation method and the filter selectivity is improved by using pump-splitting dual-stage configuration. Finally a nearly ideal rectangular MPF with flexible tenability on bandwidth, selectivity and central frequency have been demonstrated.

\section{ACKNOWLEDGMENTS}

This work is supported by 973 Program (2012CB315602), National Nature Science Foundation of China (61322507 and 61132004) and Program of Excellent PhD in China (201155).

\section{REFERENCES}

[1] J. Yao, J. Lightwave Technol. 27, 314-335 (2009).

[2] Y. Dai and J. Yao, Opt. Express 16, 4713-4718 (2008).

[3] V.R. Supradeepa et al., Nature Photonics 6, 186-194 (2012).

[4] T. Huang et al., Opt. Express 19, 6231-6242 (2011).

[5] S. Xiao and A.Weiner, J. Lightwave Technol. 24, 2523-2529 (2006).

[6] T. Tanemura et al., Opt. Lett. 27, 1552-1554 (2002).

[7] A. Zadok et al., J. Lightwave Technol. 25, 2168-2174 (2007).

[8] T. Sakamoto et al., Opt. Express 16, 8026-8032 (2008).

[9] W. Wei et al., OFC'14, W4F.5 (2014).

[10] W. Wei et al., ECOC'14, P.1.7 (2014).

[11] W. Wei et al., submitted to PTL (2015).

[12] Y. Stern et al., Photonics Research 2, B18-B25 (2014).

[13] L. Yi et al., OFC'2015, Tu3F.5 (2015). 